\title{
Loss of Gear Teeth
}

\author{
W. A. T U P L I N
}

Sheffield, England.

Gears are a very widely-used element of machinery and many millions of them continue in service without giving any trouble. On the few occasions when difficulty arises, the cause can usually be found quite quickly. Now and again, however, something happens that at first seems to defy explanation and may indeed continue to do so, but it is usually possible to identify something that might be a contributory cause. An extraordinary one is described below.

The installation concerned was one in which a large synchronous induction motor drives two centrifugal blowers through speed-increasing gears of the single-helical type. One wheel about 54 inches in diameter ran at $1,500 \mathrm{rpm}$ and drove two pinions, one at $6,650 \mathrm{rpm}$ and the other pinion at $10,250 \mathrm{rpm}$. It was in the last-named gear that the trouble seems to have originated. The main dimensions were

$\begin{array}{llccc} & & \text { Gear Wheel } & \text { Larger pinion } & \text { Smaller pinion } \\ \text { Diameter (in) } & d & 54 & 12.2 & 7.9 \\ \text { Spaed (rpm) } & n & 1500 & 6650 & 10250 \\ \text { Number of teeth } & t & 321 & 76 & 47 \\ t d n & 26 \times 10^{6} & 6.2 \times 10^{6} & 3.8 \times 10^{6} \\ \text { Centrifugal acceleration } & 1700 \mathrm{~g} & 7500 \mathrm{~g} & 1.1500 \mathrm{~g} \\ \text { at the teeth } & & & \end{array}$

The speed of the teeth relative to the common plane of the axes of the gears was about 21,000 feet per minute and this is very much higher than the average for gears of all types but not exceptional in what may be called 'high speed gears' such as are widely employed in driving centrifugal compressors or being driven by steam or gas turbines.

The gears ran continuously for 24 hours every day and (ideally) 365 days per year. Because of the nature of the driving motor, speed departed from constancy only batween the close limits maintained by the frequency of the electrical supply. 


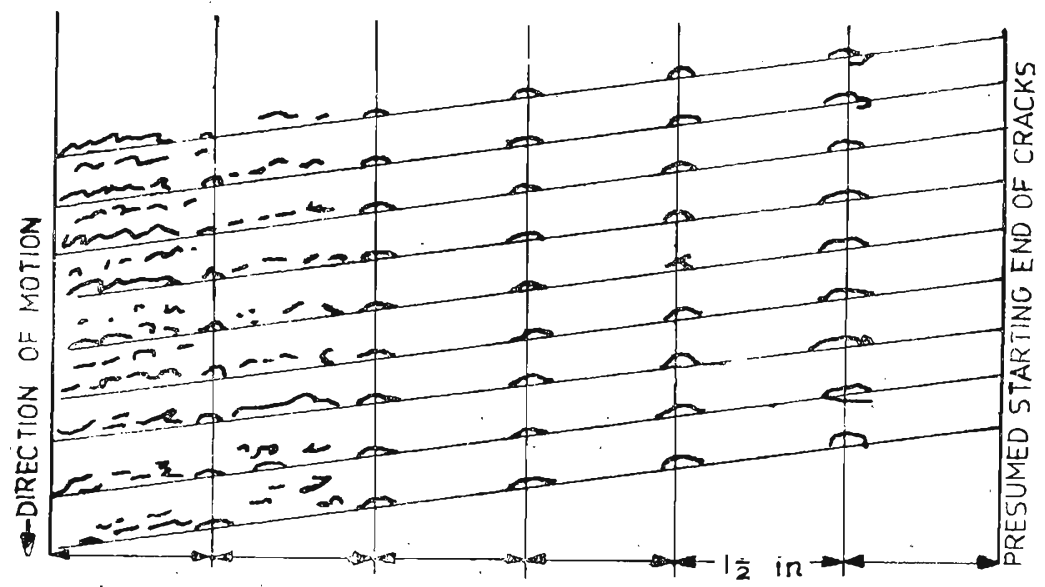

Fig. 1-Pattern of damage to the tecth of the large wheel.

Any gears of this size and speed make noise while they are running and the men in charge of the plant become accustomed to the noise and are sensitive to any quick change in it. This is specially the case where the running speed does not vary and where there are only small changes in the load. The gears concerned in this episode were noticed to have become distinctly noisier and over some 10 hours the noise increased progressively after a succession of periods of a few minutes during which loud noise was accompanied by noticeable vibration. When the vibration during one such period had developed into a hammering, it was decided that the plant should be shut down.

So the load was taken off the compressors and the motor disconnected from the electrical supply. The speed of rotation diminished gradually to zero in about 6 minutes which was normal for the installation, but a decidedly abnormal feature was that the small pinion ceased to rotate while the other gears were still running slowly. When the gear-box had been opened it was found that all the teeth had been stripped from the small pinion and that it could be rotated by hand because it was not touching the teeth of the wheel at all. It had to be concluded that the pinion had effective teeth immediately before shut-down, but lost them during run-down.

Every tooth of the wheel was found to be damaged over a width of about half an inch in each of five annuli with an axial spacing of about $1 \frac{1}{2}$ inches. The damage was mostly to the crestṣ of the teeth and did not extend very far into the depth of the teeth; it was remarkably consistent on all the teeth in any one annulus of damage. Such consistency is not uncommon in certain 
types of gear-failure as if there is no common factor in the numbers of teeth of the mating gears, any piece of broken tooth that becomes jammed between adjacent teeth of either gear acts equally to damage all the teeth of the mating gear.

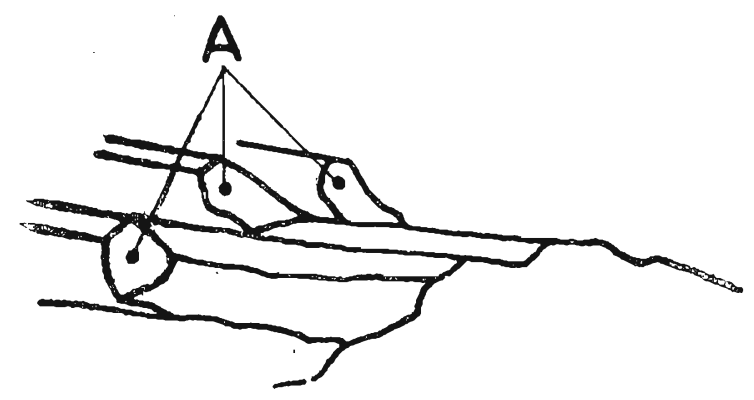

Fig. 2-Nature of fractures of pinion at intermediate stage.

The two annuli adjacent to one side of the wheel showed considerably more damage than the others, and moreover there was some damage to parts of the wheel teeth between them. Damaged areas were, in general, smooth as if from prolonged running in repeated contact with some malformation of the mating teeth. There were, however, some areas in which the teeth appeared to have flaked off in a brittle manner.

All that the body of the small pinion retained of its original 47 teeth were an equal number of stumps so short that they cleared the tips of the wheel teeth. The fractured surfaces were in general concave, were matt in appearance rather than smooth, and showed divisions corresponding to the annuli of damage on the teeth of the wheel.

From the bottom of the gear box some 250 pieces of broken pinion teeth were retrieved. They were not accurately counted as in view of their number it was perhaps thought that it did not matter if a few were lost or kept as curios. It seems reasonable to assume, however, that the original number was $47 \times 6=$ 282 , corresponding to the distinguishable divisions of fracture-areas on the body of the pinion.

The root-surfaces of most of the detached pieces of pinion-teeth were convex and had the smooth appearance characteristic of a creeping fracture whereas most of the corresponding surfaces on the body of the pinion were matt. About 30 of the pieces of pinion teeth, however, had fractures of concave form and of granulated appearance consistent with fracture after a few applications of load that produced high bending stress. 

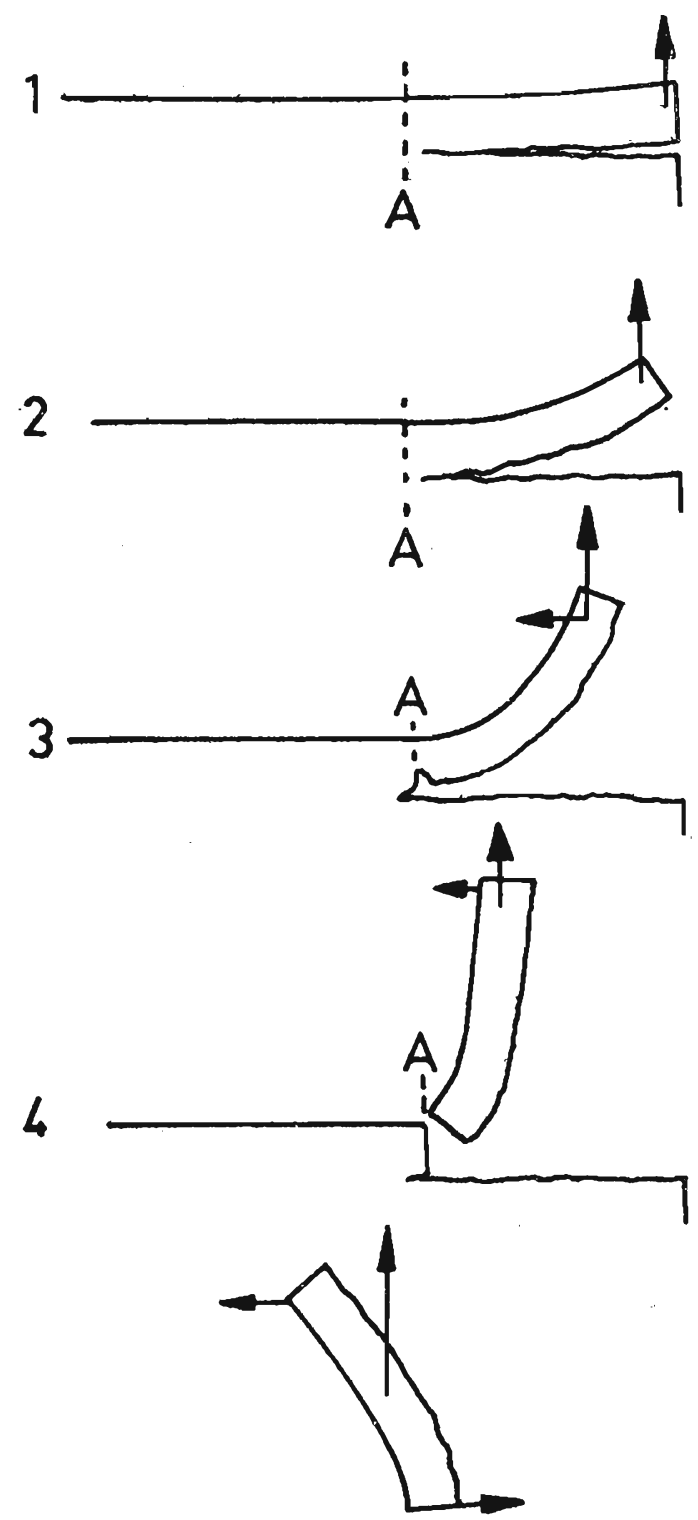

5

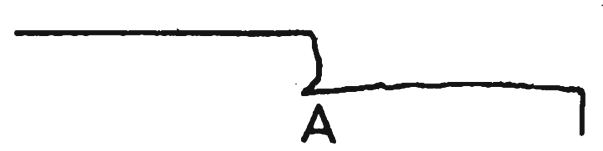

Fig. 3-Probable progress of fracture of a tooth. 
Explanation of the origin of this failure is to some extent speculative, but it is reasonable to believe that each annulus of damage corresponded to one of the short periods of specially noisy running. It is quite difficult to see why all the teeth should have failed while the tooth load corresponding to the torques applied to the gears produced a tensile stress not exceeding about 2 tons per square inch. Glossing over this, and accepting that, somehow or other, a crack was produced in the material at the root fillet at the end of a tooth, it can be recognised that such a crack would tend to extend along the root fillet and also across the thickness of the tooth to the opposite root fillet. Further extension of the crack means that a part of the tooth is held to the main body of the gear only by the other part of the same tooth and that centrifugal force on the semi-detached part of the tooth is exerting a bending moment on the crosssection of the tooth at the end of the crack. The further the crack extends, the greater the bending moment becomes; it is in fact proportional to the square of the length of the crack. This centrifugal effect is considerable as the centrifugal acceleration of the teeth of the small pinion is 11,500 times the gravitational acceleration.

Pursuing this idea numerically, it was easily found that the bending moment exerts a stress equal to the ultimate tensile strength of the material when the crack has extended to a length of about $1 \frac{1}{2}$ inches and in fact the broken pieces of pinion teeth had an average length of this amount.

Detachment of a part of a tooth in this way leaves a bent stump at the fractured end of the part of the tooth that remains attached to the body of the gear, and until this stump has been hammered down by repeated contact with the teeth of the wheel, the running is likely to be noticeably noisy. The gears still continue to run, but with a short crack extending across the width of the damaged tooth near its fractured end, and this crack gradually extends along the root of the tooth exactly as before, to produce another fracture about $1 \frac{1}{2}$ inches from its predecessor. In this way, the progressive breakage of a tooth over its whole length is readily explained.

The evidence was however, that all the teeth broke in substantialiy the same way and the implication is that they all had similar initial root-cracks although the bending stress corresponding to the applied load was far too small to start a crack in the material of the pinion.

The implication is that, right from the start, the bending stress at the root fillets was very much higher than that corresponding to the applied torque. In any circumstance of this kind in fast running machinery, the possibility of load-magnification by resonant vibration naturally occurs to any investigator. A disconcerting feature of this occurrence is that any such resonance seemed to persist even after progressive damage to the pinion had markedly altered its stiffness so that natural frequencies associated with it had also been altered, whereas the frequencies of possible origins of vibration-excitation had remained unchanged. Reflection on this encourages one to seek a type of vibration of 
such a nature that its associated natural frequency is not affected by shortening of the pinion teeth. Perhaps it is a mode of vibration whose natural frequency is determined only by the diameter of the pinion?

Confining attention, for initial simplicity, to a single transverse section of the pinion it is easy to realise that application of load to any tooth in a very short time produces at its root a momentary shear stress in the circumferential direction. A result is that a wave of shear stress travels diametrically across the circular transverse section of the root cylinder, is reflected from the vicinity of the root of the tooth diametrically opposite to the first-considered tooth and returns to it.

In steel a wave of direct stress travels at about a million feet per minute ; a wave of shear stress travels at that speed multiplied by $\sqrt{ }$ (Modulus of elasticity for shear stress/Modulus of elasticity for direct stress) and this is equal to about 0.63 .

If the reflected wave reaches the vicinity of its origin at the instant at which load is applied to a tooth adjacent to the first one, it is likely to add to the effect of the load in producing stress. Repetition of this amplification at the instants of load application to succesive teeth produces the cumulative effect of resonant vibration restrained only by the small camping by hysteresis in the steel. In these circumstances, a magnification of the order of 200 is possible and this is far more than sufficient to start a crack at every tooth.

A little arithmetic applied tc quantify this concept leads to the conclusion that resonance of this kind is possible if $t d n$, the product of pinion speed, pinion diameter and number of teeth in the pinion, is about $3.8 \times 10^{6}$. The figures for the pinion that failed have almost exactly this product.

If this type of resonance was in fact the origin of the dangerously high stress in this pinion one may conclude that sheer bad luck, in conjunction with the constancy of speed of a synchronous induction motor, was the origin of this very spectacular failure.

In a gear in the form of a thin ring, transmission of a wave of shear stress across a diameter is clearly impossible but transmission of a wave of direct stress round the ring can occur. It is extremely interesting to note that the ratio of the speeds of propagation of the two types of wave is almost exactly $2 / \pi$ the ratio of the lengths of the paths to be followed by the waves in returning to the starting point, and so the critical value of $t d n$ is the same for both types of stress. Hence it is reasonable to conclude that whether a steel gear is a solid cylinder or a thin ring or is a solid of revolution of any intermediate form, it is desirable to avoid running it at the speed that gives $t d n$ any value between about $3.6 \times 10^{6}$ and $4 \times 10^{6}$, lest there should be a recurrence of the type of resonant vibration that may have been a contributory cause of the extraordinary gear failure described here. 